\title{
Moscas frugívoras (Diptera, Tephritoidea) coletadas em Aquidauana, MS
}

\author{
Sérgio Roberto Rodrigues ${ }^{1}$; Lidiane Rodrigues Nantes ${ }^{1}$; Silvia Regina de Souza ${ }^{1}$; Alfredo Raul Abot ${ }^{1}$ \\ \& Manoel A. Uchôa-Fernandes ${ }^{2}$
}

\begin{abstract}
${ }^{1}$ Universidade Estadual de Mato Grosso do Sul (UEMS), Rodovia Aquidauana/CERA, km 12, 79200-000 Aquidauana-MS. sergio@uems.br ${ }^{2}$ Laboratório de Insetos Frugívoros, Caixa Postal 241, Faculdade de Ciências Biológicas e Ambientais, Universidade Federal da Grande Dourados (UFGD), Rod. Dourados Itahum, Km 12, 79804-970 Cidade Universitária, Dourados-MS. uchoa@ceud.ufms.br
\end{abstract}

\begin{abstract}
Frugivorous flies (Diptera, Tephritoidea) collected in Aquidauana, MS. In Brazil, frugivorous flies represent important pests of fruits and vegetables. Information on populational fluctuation of these species in each biome is an important requirement for the adoption of a strategy of pest control in the agroecosystems. The objective of this paper is to assess the diversity of fruit fly species occurring in wild and cultived fruits in Aquidauana, MS. Twenty-nine fruit species were sampled from February 2003 to January 2004. The Tephritidae species recovered were: Anastrepha striata Schiner, 1868, Anastrepha obliqua (Macquart, 1835) and Ceratitis capitata (Wiedemann, 1824). The frugivorous Lonchaeidae and Muscidae recovered were: Neosilba sp. and Atherigona orientalis (Schiner, 1868), respectively. A total of 2.568 flies were obtained, from which 2.394 flies were the Mediterranean fruit fly, C. capitata. The association between frugivorous flies and the fruit species is discussed.
\end{abstract}

KEYWORDS. Anastrepha; Brazil; Ceratitis capitata; Lonchaeidae; Tephritidae.

RESUMO. Moscas frugívoras (Diptera, Tephritoidea) coletadas em Aquidauana, MS. No Brasil as moscas frugívoras são pragas importantes de frutas e hortaliças. O conhecimento da flutuação populacional dessas espécies em cada bioma é um importante requisito para a adoção de estratégia de controle de pragas nos agroecossistemas. O objetivo desse trabalho foi avaliar a diversidade de espécies de moscas-das-frutas infestantes de frutas silvestres e cultivadas em Aquidauana, MS. Vinte e uma espécies de frutas foram amostradas de fevereiro de 2003 a janeiro de 2004. As espécies de Tephritidae encontradas foram: Anastrepha striata Schiner, 1868, Anastrepha obliqua (Macquart, 1835) e Ceratitis capitata (Wiedemann, 1824). Os frugívoros Lonchaeidae e Muscidae encontrados foram: Neosilba sp. e Atherigona orientalis (Schiner, 1868), respectivamente. Um total de 2.568 moscas foram coletadas, das quais 2.394 representadas pela moscado-Mediterrâneo C. capitata. A associação entre moscas frugívoras e espécies de frutas é discutida.

PALAVRAS-CHAVE. Anastrepha; Brasil; Ceratitis capitata; Lonchaeidae; Tephritidae.

No Estado de Mato Grosso do Sul (MS), a fruticultura representa importante fonte de renda para várias propriedades rurais. Segundo dados do IBGE (2000), várias são as culturas exploradas economicamente com as seguintes áreas cultivadas: banana (3.198 ha), abacaxi (183 ha), goiaba (16 ha), laranja (520 ha), limão Taiti (68 ha), tangerina Poncã (156 ha), mamão (31 ha), maracujá (22 ha), uva (90 ha), melancia (917 ha) e melão (16 ha). Outra importante frutífera é a manga com 147 ha (Agrianual 2002).

Dentre os principais fatores que limitam a produção de frutíferas, destacam-se as moscas-das-frutas, as quais podem causar sérios danos, sendo a família Tephritidae a maior e economicamente mais importante. É composta por 500 gêneros e mais de 4.000 espécies descritas. Aproximadamente $35 \%$ delas atacam frutos, sendo que representantes dos gêneros Bactrocera, Ceratitis, Dacus, Anastrepha e Rhagoletis estão entre as pragas mais importantes da fruticultura mundial (White \& Elson-Harris 1994). No Brasil, segundo Zucchi (2000), as espécies de maior importância econômica são as do gênero Anastrepha Schiner, 1868 e Ceratitis capitata (Wiedemann 1824).
Na região Centro-Oeste do Brasil, estes dípteros frugívoros representam um dos principais fatores de perdas da produção frutícola (Uchôa-Fernandes et al. 2003a, Veloso et al. 1994). Além de Tephritidae, espécies de alguns gêneros de Lonchaeidae têm se destacado como pragas importantes em frutíferas cultivadas (Raga et al. 1996; Norrbom \& McAlpine 1997).

Apesar do grande potencial para fruticultura no MS e da importância econômica dos tefritóideos para as frutíferas tropicais, há poucos registros sobre as espécies de moscas ocorrentes (Malavasi \& Morgante 1980; Uchôa-Fernandes \& Zucchi 1999, 2000; Zucchi 2000).

O presente trabalho teve por objetivo estudar as espécies de moscas frugívoras e seus frutos hospedeiros nas proximidades da cidade de Aquidauana, MS.

O trabalho foi desenvolvido na Fazenda Experimental da Universidade Estadual de Mato Grosso do Sul (UEMS). Os frutos foram amostrados em área de mata de Cerrado e na área de citricultura (pomar da UEMS) e, de frutos coletados na cidade de Aquidauana/MS. Estes foram colhidos no período de fevereiro de 2003 a janeiro de 2004 e acondicionados no laboratório. 
Tabela I. Espécies frutíferas, quantidade total de frutos (g) amostrados e abundância de dípteros em Aquidauana, MS (fevereiro de 2003 a janeiro de 2004).

\begin{tabular}{|c|c|c|c|c|c|}
\hline Espécies de frutiferas & Quantidade (g) & Espécies de Diptera & $\mathrm{N}^{\mathrm{o}}$ de adultos & Espécies de frutíferas & ntidade $(g)$ \\
\hline Caraguatá (Bromelia sp) & $5.038,27$ & Otitidae & 08 & Guavira (Campomanesia sessiflora) & $1.658,22$ \\
\hline Ingá (Inga laurina) & $1.935,98$ & Neosilba sp. & 01 & Jabuticaba (Myrciaria jabuticaba) & $8.628,85$ \\
\hline Goiaba (Psidium guajava) & $20.900,24$ & A.striata & 12 & Jambolão (Syzygium sp.) & $3.863,47$ \\
\hline Laranja (Citrus sinensis) & $38.556,73$ & $\begin{array}{l}\text { A.orientalis } \\
\text { C. capitata }\end{array}$ & $\begin{array}{l}06 \\
01\end{array}$ & Limão (Citrus limon) & $4.630,11$ \\
\hline Poncã (Citrus reticulata) & $6.557,34$ & $\begin{array}{l}\text { A.orientalis } \\
\text { Otitidae }\end{array}$ & $\begin{array}{l}06 \\
01\end{array}$ & Maracujá amarelo (Passiflora edulis) & $16.594,15$ \\
\hline Cirigüela (Spondias purpurea) & $11.218,42$ & $\begin{array}{l}\text { A. obliqua } \\
\text { Anastrepha sp. }\end{array}$ & $\begin{array}{l}81 \\
57\end{array}$ & Mamão (Carica papaya) & $2.623,34$ \\
\hline Sete copas (Terminalia catappa) & $17.950,28$ & $\begin{array}{l}\text { C. capitata } \\
\text { Neosilba } \mathrm{sp} .\end{array}$ & $\begin{array}{c}2.393 \\
02\end{array}$ & Melancia (Cucumes sp.) & $2.000,05$ \\
\hline Abacate (Persea americana) & $5.016,28$ & - & - & Manga Borbon (Mangifera indica) & $9.099,69$ \\
\hline Acerola (Malpighia punicifolia) & $6.239,68$ & - & - & Manga espada (Mangifera indica) & $3.126,00$ \\
\hline Amora (Morus) & 294,32 & - & - & Manga rosa (Mangifera indica) & $1.556,50$ \\
\hline $\begin{array}{l}\text { Água pomba (Melicoccus } \\
\text { lepidopetalus) }\end{array}$ & $7.255,69$ & - & - & Manga margarita (Mangifera indica) & $5.482,74$ \\
\hline Araçá (Psidium sp.) & 157,92 & - & - & Oiti (Licania sp.) & $12.141,46$ \\
\hline Ata (Annona squamosa) & $2.888,88$ & - & - & Pequi (Caryocar brasiliense) & $1.022,12$ \\
\hline Caju (Anacardium occidentale) & 117,00 & - & - & Tamarino (Tamarindus indica) & 736,69 \\
\hline Genipapo (Genipa americana) & 485,14 & - & - & & \\
\hline
\end{tabular}

$\mathrm{Na}$ área de Cerrado, semanalmente foram realizadas coletas de frutos das espécies nativas e cultivadas. Os frutos de citros foram obtidos do pomar ao longo dos ciclos de frutificação. Em Aquidauana procedeu-se à coleta apenas de frutos de Terminalia catappa (Sete-copas). Para cada espécie vegetal procurou-se coletar o mínimo de $10 \mathrm{~kg}$ de frutos, entretanto, nem sempre isso foi possível (Tabela I).

Após coletados e pesados, os frutos foram mantidos em camada única, sobre suporte telado com malha de $5 \mathrm{~mm}$, em bandejas de plástico (55x45×35 $\mathrm{cm}$ de profundidade), com uma lâmina de água de cerca de $15 \mathrm{~mm}$ para a retenção das larvas de último ínstar que abandonavam os frutos para empupar. $\mathrm{O}$ material foi mantido em laboratório sob temperatura ambiente. A cada $12 \mathrm{~h}$, as larvas eram recolhidas das bandejas e acondicionadas em potes de acrílico transparentes $(300 \mathrm{~mL})$ sobrepostos, assumindo a forma de um barril, contendo areia estéril umedecida em água destilada, sendo que as formas jovens permaneceram nesses recipientes até a emergência dos adultos (Uchôa-Fernandes \& Zucchi 1999).

Os frutos foram mantidos por 30 a 40 dias, até que todos os insetos empupassem. Os adultos provenientes de cada coleta, foram fixados em álcool $70 \%$ e mantidos em frascos rotulados, para a posterior identificação (Uchôa-Fernandes \& Zucchi 1999).

Exemplares identificados das moscas-das-frutas foram depositados na coleção Zoológica da Universidade Federal de Mato Grosso do Sul, em Campo Grande, MS.

Foram coletados frutos de 29 espécies, sendo obtidas moscas apenas de sete delas: Bromelia sp. (Caraguatá), Inga laurina (Ingá), Psidium guajava (Goiaba), Citrus sinensis (Laranja), Citrus reticulata (Poncã), Spondias purpurea (Cirigüela) e T. catappa (Sete-copas) (Tabela I). Uchôa-
Fernandes et al. (2002) ao realizarem coletas de frutos de 35 espécies em áreas de Cerrado no MS, obtiveram moscas-dasfrutas de 29 plantas.

As moscas-das-frutas coletadas pertencem às famílias Tephritidae, Lonchaeidae e Otitidae, além de moscas decompositoras da família Muscidae. De Tephritidae foram obtidas: Anastrepha striata Schiner, 1868, A. obliqua (Macquart, 1835) e C. capitata; de Lonchaeidae Neosilba sp. e, de Muscidae, Atherigona orientalis (Schiner, 1868) (Tabela I).

A espécie mais abundante foi C. capitata, pois das 2.568 moscas-das-frutas coletadas, 2.394 adultos foram recuperados de laranja e sete-copas, representando $93,22 \%$ do total de moscas frugívoras (Tabela I). Uchôa-Fernandes et al. (2003a) encontraram, também, C. capitata como o tefritídeo dominante, no MS.

Anastrepha obliqua foi mais abundante (81 exemplares), representando $54 \%$ dos espécimes desse gênero e $A$. striata (12 espécimes) representou $8 \%$ dos indivíduos (Tabela I).

Coletas de frutos realizadas por Uchôa-Fernandes et al. (2002), em alguns municípios do MS, encontraram dez espécies de Anastrepha: A. fraterculus (Wied., 1830), A. grandis (Macquart, 1846), A. montei Lima, 1934, A. obliqua, A. pickeli Lima, 1934, A. sororcula Zucchi, 1979, A. striata, A. turpiniae Stone, 1942, A. zenildae Zucchi, 1979 e Anastrepha sp. Nesse estudo, $A$. sororcula foi a mais abundante com 1.315 espécimes coletados, seguido por $A$. obliqua com 951 espécimes e $A$. turpiniae com 165 indivíduos.

Analisando-se por espécie vegetal foi verificado que, de frutos de S. purpurea foram obtidas 138 moscas-das-frutas, sendo 81 espécimes de A. obliqua e 57 de Anastrepha sp., provavelmente co-específicos (Tabela I). Esses dípteros ocorreram em fevereiro e março (Tabela II). Dos frutos dessa 
Tabela II. Espécies de plantas e de moscas frugívoras amostradas em Aquidauana, MS (fevereiro de 2003 a janeiro de 2004).

\begin{tabular}{|c|c|c|c|c|c|c|c|}
\hline \multirow[b]{2}{*}{$\begin{array}{l}\text { Mês de } \\
\text { ocorrência }\end{array}$} & \multicolumn{7}{|c|}{ Espécies de frutíferas } \\
\hline & $\begin{array}{l}\text { Citrus } \\
\text { sinensis } \\
\text { Laranja }\end{array}$ & $\begin{array}{l}\text { Terminalia } \\
\text { catappa } \\
\text { Sete copas }\end{array}$ & $\begin{array}{l}\text { Bromelia } \\
\text { sp. } \\
\text { Caraguatá }\end{array}$ & $\begin{array}{l}\text { Psidium } \\
\text { guajava } \\
\text { Goiaba }\end{array}$ & $\begin{array}{l}\text { Inga } \\
\text { laurina } \\
\text { Ingá }\end{array}$ & $\begin{array}{l}\text { Spondias } \\
\text { purpurea } \\
\text { Cirigüela }\end{array}$ & $\begin{array}{l}\text { Citrus } \\
\text { reticulata } \\
\text { Poncã }\end{array}$ \\
\hline Fevereiro & & & & & 1 Neosilba sp. & $\begin{array}{l}76 \text { A. obliqua } \\
43 \text { Anastrepha } \mathrm{sp} .\end{array}$ & \\
\hline Março & & & & & & $\begin{array}{l}14 \text { Anastrepha sp. } \\
5 \text { A. obliqua }\end{array}$ & \\
\hline Abril & & & 8 Otitidae & & & & \\
\hline Maio & 3 A. orientalis & & & & & & $\begin{array}{l}6 \text { A. orientalis } \\
1 \text { Otitidae }\end{array}$ \\
\hline Junho & & 109 C. capitata & & & & & \\
\hline Julho & 1 C. capitata & 723 C. capitata & & 12 A. striata & & & \\
\hline Agosto & & 608 C. capitata & & & & & \\
\hline Setembro & & $\begin{array}{l}886 \text { C. capitata } \\
2 \text { Neosilba } \mathrm{sp} .\end{array}$ & & & & & \\
\hline Outubro & $3 \mathrm{~A}$. orientalis & 67 C. capitata & & & & & \\
\hline Total & 7 & 2.395 & 8 & 12 & 1 & 138 & 7 \\
\hline
\end{tabular}

mesma espécie coletados nos municípios de Aquidauana, MS, Anastácio, MS e Terenos-MS, Uchôa-Fernandes et al. (2003b) obtiveram 99 exemplares de $A$. obliqua, 2 de A. sororcula, 1 de A. turpiniae, 226 de Anastrepha spp., 3 de C. capitata, 1 de Neosilba sp. e 1 de Dasiops sp.

Em T. catappa, foram obtidos 2.395 dípteros: 2.393 espécimes de $C$. capitata, representando 99,91\% das moscas e 02 espécimes de Neosilba sp. (0,09\%) (Tabela I). C. capitata ocorreu de junho a outubro (Tabela II), colonizando frutos de sete-copas coletados em Aquidauana, MS. Também de setecopas, Uchôa-Fernandes et al. (2003b) coletaram 3 espécimes de Anastrepha spp., 1 de $A$. zenildae e 2.131 de $C$. capitata. Cerca de $99 \%$ dos adultos coletados, são de C. capitata, comprovando a predominância dessa espécie em frutos de $T$. catappa.

Canal et al. (1998) verificaram que em Janaúba-MG, $C$. capitata representou $85,54 \%$ do total de moscas-das-frutas coletadas em área urbana. Após realizar coletas em quatro municípios do Estado de Minas Gerais, os autores concluíram que essa espécie caracteriza-se como uma praga de frutíferas em área urbana.

Das 173 moscas-das-frutas coletadas em áreas de Cerrado e de citricultura, apenas um exemplar $(0,58 \%)$ representou $C$. capitata, enquanto que de 2.395 indivíduos coletados em ambiente urbano, 2.393 (99,91\%) pertenciam a essa espécie (Tabela I).

Em frutos de $C$. sinensis foram encontradas $C$. capitata $\mathrm{e}$ A. orientalis (Tabela I). Esta última coletada em maio e outubro e, C. capitata em Julho (Tabela II). De frutos de $C$. reticulata foram encontradas em maio; 6 exemplares de $A$. orientalis e um de Otitidae (Tabelas I e II). Não foram coletados de frutos cítricos Neosilba sp., entretanto, de pomares cítricos em MS cerca de 5.966 exemplares dessa espécie foram coletados por Uchoa-Fernandes et al. (2003c).

Inventários de moscas-das-frutas realizados com armadilhas McPhail em pomares cítricos nos municípios de Anastácio, MS e Terenos, MS, por Uchôa-Fernandes et al. (2003c), amostraram 22 espécies de Anastrepha: A. alveatoides Blanchard, 1961, A. bezzii Lima, 1934, A. castanea Norrbom, 1998, A. daciformis Bezzi, 1909, A. dissimilis Stone, 1942, A. distincta Greene, 1934, A. fraterculus, A. grandis, $A$. haywardi Blanchard, 1937, A. leptozona Hendel, 1914, A. macrura Hendel, 1914, A. montei, A. obliqua, A. pickeli, A. punctata Hendel, 1914, A. rheediae Stone, 1942, A. serpentina (Wied., 1830), $A$. sororcula, A. striata, A. turpiniae, A. undosa Stone, 1942, A. zenildae e três Anastrepha sp., além de 1 exemplar de $C$. capitata, 2 de Dasiops sp., 1 de Lonchaea sp. e 1 de Neosilba sp. De frutos cítricos coletados em São Paulo por Malavasi \& Morgante (1980) foram encontrados adultos de Neosilba sp. (como Silba).

De frutos de $P$. guajava foram encontrados 12 espécimes de $A$. striata, em julho (Tabelas I e II). Segundo Nascimento et al. (1982) na região do Recôncavo Baiano, em frutos de goiaba a espécie mais comum foi $A$. fraterculus. Uchôa-Fernandes et al. (2002) coletaram dessa frutífera, A. sororcula, A. turpinae, A. striata, A. obliqua, A. zenildae, A. fraterculus, C. capitata e Neosilba spp. No semi-árido do Brasil, Araújo et al. (1996) relataram que $A$. zenildae tem sido a espécie mais comum em $P$. guajava. Dessa mirtácea, em área urbana do município de Ouro Preto do Oeste, RO, Ronchi-Teles \& Silva (1996) encontraram C. capitata.

De I. laurina foi obtido um adulto de Neosilba sp. em fevereiro/2003 (Tabelas I e II). Dessa mesma frutífera, em alguns municípios do MS, Uchôa-Fernandes et al. (2002) encontraram 50 espécimes de $C$. capitata e 199 de Neosilba spp.

De Bromelia sp., foram obtidos 8 adultos da família Otitidae, em abril/2003 (Tabelas I e II). Dessa família, 108.659 indivíduos capturados em armadilhas McPhail, sendo que esse número representou mais de $92 \%$ do total de insetos capturados nas armadilhas iscadas com atrativo alimentar. 
De algumas frutas coletadas como, jabuticaba e araçá, não foram encontradas moscas-das-frutas (Tabela I). Dessas frutas Uchôa-Fernandes et al. (2002) coletaram várias espécies de Anastrepha em MS.

\section{REFERÊNCIAS}

Agrianual. 2002. Anuário da agricultura brasileira. São Paulo: FNP Consultoria e Comércio. 536p.

Araújo, E. L.; R. A. Zucchi; A. Malavasi \& J. Negreiros. 1996. Levantamento de moscas-das-frutas do gênero Anastrepha Schiner, 1868 (Dip., Tephritidae) nos municípios de Mossoró e Assú-RN. Revista de Agricultura 71: 225-232.

Canal, N. A.; C. D. Alvarenga \& R. A Zucchi. 1998. Análise faunística de espécies de moscas-das-frutas (Dip., Tephritidae) em Minas Gerais. Scientia Agricola 55: 15-24.

IBGE. Censo Populacional de 2000. disponível em: http:// www.ibge.gov.br/home/estatistica/ população/ censo2000/ defaulttab munic.shtm. Acesso em abril de 2005.

Malavasi, A. \& J. S. Morgante. 1980. Biologia de mosca-das-frutas (Diptera, Tephritidae). II: índices de infestação em diferentes hospedeiros e localidades. Revista Brasileira de Biologia 40: $17-24$.

Nascimento, A. S.; R. A. Zucchi, J. S. Morgante \& A. Malavasi. 1982. Dinâmica populacional das moscas-das-frutas do gênero Anastrepha (Diptera: Tephritidae) no Recôncavo Baiano II - Flutuação Populacional. Pesquisa Agropecuária Brasileira 17: 969-980.

Norrbom, A. L. \& J. F. McAlpine. 1997. A revision of the neotropical species of Dasiops Rondani (Diptera, Lonchaeidae) attacking Passiflora (Passifloraceae). Memmoirs of the Entomological Society of Washington 18: 189-211.

Raga, A.; M. F. Souza Filho; V. Arthur \& A. L. M. Martins. 1996. Avaliação da infestação de moscas-das-frutas em variedades de café (Coffea spp.). Arquivos do Instituto Biológico 63: 59-63.

Ronchi-Teles, B. \& N. M Silva. 1996. Primeiro registro de ocorrência da mosca-do-mediterrâneo, Ceratitis capitata (Wied.) (Diptera:
Tephritidae) na Amazônia Brasileira. Anais da Sociedade Entomológica do Brasil 25: 569-670.

Uchôa-Fernandes., M. A. \& R. A Zucchi. 1999. Metodologia de colecta de Tephritidae y Lonchaeidae frugívoros (Diptera, Tephritoidea) y sus parasitoides (Hymenoptera). Anais da Sociedade Entomológica do Brasil 28: 601-610.

Uchôa-Fernandes, M. A. \& R. A. Zucchi. 2000. Moscas-das-frutas nos Estados Brasileiros. Capítulo 35. Mato Grosso e Mato Grosso do Sul. P. 241-245. In: Malavasi, A. \& Zucchi, R. A. Moscas-dasfrutas de Importância Econômica no Brasil: Conhecimento Básico e Aplicado. Ed. Holos-FAPESP. 327p.

Uchôa-Fernandes, M. A.; I. Oliveira; R. M. S. Molina \& R. A Zucchi 2002. Species diversity of frugivorous flies (Diptera: Tephritoidea) from hosts in the cerrado of the State of Mato Grosso do Sul, Brazil. Neotropical Entomology 31: 515-524.

Uchôa-Fernandes, M. A.; I. Oliveira; R. M. S. Molina \& R. A. Zucchi. 2003a. Populational fluctuation of frugivorous flies (Diptera: Tephritoidea) in two orange groves in the state of Mato Grosso do Sul, Brazil. Neotropical Entomology 32: 19-25.

Uchôa-Fernandes, M. A.; R. M. S. Molina; I. Oliveira; R. A. Zucchi; N. A. Canal \& N. B. Diaz. 2003b. Larval endoparasitoids (Hymenoptera) of frugivorous flies (Diptera, Tephritoidea) reared from fruits of the cerrado of the State Mato Grosso do Sul, Brazil. Revista Brasileira de Entomologia 47: 181-186.

Uchôa-Fernandes, M. A.; I. Oliveira; R. M. S. Molina \& R. A. Zucchi 2003c. Biodiversity of frugivorous flies (Diptera, Tephritoidea) captured in citrus groves, Mato Grosso do Sul, Brazil. Neotropical Entomology 32: 239-246.

Veloso, V. R. S.; P. M. Fernandes; M. R. Rocha; M. V. Queiroz \& R. M. R. Silva. 1994. Armadilhas para o monitoramento e controle das moscas-das-frutas Anastrepha spp. e Ceratitis capitata (Wied.) Anais da Sociedade Entomológica do Brasil 23: 487-493.

White, I. M. \& M. M. Elson-Harris. 1994. Fruit flies of economic significance: their identifications and bionomic. Wallinford: CAB International. $601 \mathrm{p}$.

Zucchi, R. A. 2000. Taxonomia. In: Malavasi, A. \& R. A. Zucchi Moscas-das-frutas de Importância Econômica no Brasil: Conhecimento Básico e Aplicado. Ed. Holos-FAPESP. 327p.

Recebido em 15/06/2005; aceito em 05/12/2005 